\title{
Comparison of Spinal Nerve Root Block Alone and Spinal Nerve Root Block with Psoas Compartment Block in Patients with a Herniated Lumbar Disc
}

\author{
Sung Mo Ryu', Seung-Kook Kim ${ }^{2 \bowtie u n-S a n g ~ K i m}{ }^{1}$, Soo-Chan Lee ${ }^{2}$ \\ ${ }^{1}$ Department of Neurosurgery Spine Center, Samsung Medical Center, Sungkyunkwan University School of Medicine, Seoul, Korea \\ ${ }^{2}$ Department of Spine Center Himchan Hospital, Incheon, Korea
}

\begin{abstract}
Objective: Spinal nerve root blocks (SNRBs) have been used to provide pain control for herniated lumbar discs (HLDs). Psoas compartment blocks (PCBs) have been used to provide pain control for lumbar sprain, and analgesia for post-operative pain. This block is advocated for relieving pain of various origins in the lower extremities and pelvic region. However, the efficacy of additional PCB is unknown. In this study, we compared conventional SNRB versus SNRB with PCB for HLDs. Methods: Forty patients with HLD disease were randomly divided into two groups. Group A was administered dexamethasone $(2 \mathrm{~mL})$ with lidocaine $(2 \mathrm{~mL}, 0.5 \%)$ into the transforaminal epidural space $(\mathrm{L} 4-\mathrm{L} 5, \mathrm{~L} 5-\mathrm{S} 1)$ in the prone position. Group B was administered lidocaine $(10 \mathrm{~mL}, 0.5 \%)$ to the bilateral psoas compartment muscles ( $L 3-L 4$, total: $20 \mathrm{cc}$ ) and was administered an SNRB using the same regimen as described above. Results: Neither method failed in any of the patients in either group. Initial visual analogue scale (VAS) scores were decreased, although not statistically significantly, in group B as compared to group A. By 60 days post-operatively, VAS scores were decreased in both groups, but also not statistically significantly. Conclusion: SNRB is a proven treatment for controlling inflammation in HLD patients. Use of PCB blocks may extend the initial pain control by providing muscular stabilization. However, SNRB with PCB was not more effective than conventional SNRB.
\end{abstract}

Key Words: Epidural space; Intervertebral disc displacement; Back pain

$\triangle$ Corresponding Author: Seung-kook Kim, Department of Spine Center, Incheon Himchan Hospital, 118 Yongdam-ro, Yunsoo-gu, Incheon 21927, Korea. Tel: +82-1899-2220, 3491, Fax: +82-2-3410-0048, E-mail: deux8888@naver.com

\section{INTRODUCTION}

Herniated lumbar disc (HLD) disease is one of the most common causes of back pain. Spinal nerve root blocks (SNRBs) and median branch blocks (MBBs) have long been used to provide pain control for herniated lumbar discs (HLDs) ${ }^{7}$. Psoas compartment blocks (PCBs) have been used to provide pain control for lumbar sprain, and analgesia for post-operative pain ${ }^{3)}$. Back pain in patients has a complex pathophysiology, such as muscle sprain, neurogenic pain, and degenerative changes. Additionally, patients often desire to avoid unnecessary surgery.

If an additional PCB can be more efficient in reducing back pain, in-hospital stay can be reduced, resulting in medical service cost-savings. Currently, this block is advocated for relieving pain of various origins in the lower extremities and pelvic region. However, the efficacy of additional PCB for the relief of pain associated with HLDs is unknown. Therefore, in this study, we compared conventional SNRB versus SNRB with PCB in patients with HLD disease.

\section{MATERIALS AND METHODS}

Patients with HLD disease were recruited from May 2013 to June 2014; patients ranged in age from 33 to 74 years. This study was a randomized controlled trial and designed case series, involving one surgeon at a single center. Patients were randomly assigned to one of two groups using a randomization sequence table produced by random allocation software by a physician who was not involved in this study. Inclusion criteria were symptoms of axial low back pain with or without unilateral radiating pain lasting more than 2 months. Patients with low back pain due to motor weakness due to complete foramen obstruction or other origins (spondylolisthesis and spondylolysis) were excluded, and we limited the HLD location to L4-L5 and L5-S1.

We divided patients into two groups based on the procedure used (Table 1). Group A constituted the conventional SNRB group (Fig. 1A); these patients were treated with an SNRB+ MBB. The SNRB involved administration of dexamethasone 
$(2 \mathrm{~mL})$ with lidocaine $(2 \mathrm{~mL}, 0.5 \%)$ into the transforaminal epidural space (L4-L5, L5-S1), with the patient in the prone position. The MBB involved administration of dexamethasone $(1 \mathrm{~mL})$ with lidocaine $(1 \mathrm{~mL}, 0.5 \%)$ into the transverse process and superior articular process junction (Burton point, Scotty dog sign). Group B was treated with an SNRB+MBB, and was given an additional bilateral PCB injection (Fig. 1B) The PCB involved administration of lidocaine $(10 \mathrm{~mL}, 0.5 \%)$ into the bilateral psoas compartment space (L3-L4, total $20 \mathrm{~mL}$ ). All procedures involved a single injection. After the procedures, 2 weeks NSAID and physical treatment was done.

The outcome measure was the visual analogue scale (VAS) score for back and radiating leg pain at before, post-operative day (POD) 1 and POD 60 (Table 2). Pain scales were assessed in the out-patient clinic.

Statistical analysis was performed using SPSS software

Table 1. Demographic findings in SNRB+MBB and SNRB+PCB groups

\begin{tabular}{llccc}
\hline & & SNRB+MBB & SNRB+PCB & p-value \\
\hline Age (years) & & $43.4 \pm 13.2$ & $53.4 \pm 18.8$ & $>0.05$ \\
Sex & Male & 12 & 8 & $>0.05$ \\
& Female & 8 & 12 & \\
Disc & Central & 10 & 8 & $>0.05$ \\
location & Paracentral & 10 & 12 & \\
Disc type & Shoulder & 18 & 15 & $>0.05$ \\
& Axillary & 2 & 5 & \\
Migration & Up-migrated & 2 & 1 & $>0.05$ \\
& Down-migrated & 5 & 5 & \\
& Low-grade & 2 & 3 & \\
& High-grade & 3 & 1 & \\
\hline
\end{tabular}

SNRB: spinal nerve root block; MBB: median branch block; PCB: psoas compartment block
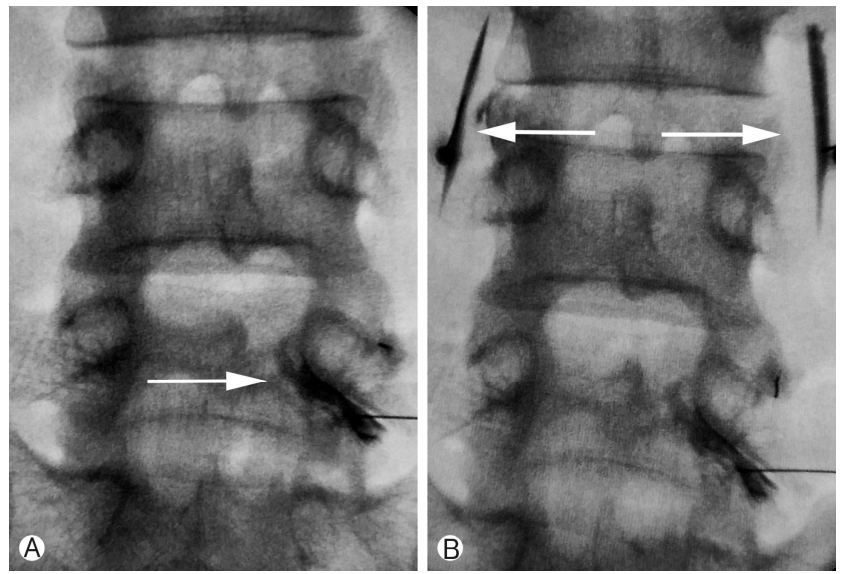

Fig. 1. (A) Group A shows conventional spinal root block (White arrow: single nerve root block and median branch block). (B) Group B shows additional psoas compartment block (PCB) group (White arrow: bilateral additional PCB).
(V22.0; SPSS, Inc., Chicago, IL, USA). Depending on the variables, intergroup differences were analyzed using independent sample t-tests and paired t-tests. Statistical significance was set at $p<0.05$.

\section{RESULTS}

Twenty patients each underwent SNRB+MBB (group A) or $\mathrm{SNRB}+\mathrm{MBB}+\mathrm{PCB}$ (group $\mathrm{B}$ ) procedures. The mean age of the patients was $43.4 \pm 13.2$ years in group $A$ and $53.4 \pm 18.8$ years in group $\mathrm{B}$ (Table 1).

At POD 1, in group A, the mean VAS scores for back and leg pain decreased from $7.7 \pm 1.0$ to $3.9 \pm 1.2$ and from $6.8 \pm 1.3$ to $1.8 \pm 1.0$, respectively. In group B, the mean VAS scores for back and leg pain decreased from $7.7 \pm 1.4$ to $2.9 \pm 2.3$ and from $6.5 \pm 1.5$ to $2.3 \pm 1.3$, respectively (Table 2 ).

At POD 60, in group A, the mean VAS scores for back and leg pain had decreased to $2.3 \pm 1.2$ and $1.3 \pm 1.2$, respectively. In group B, the mean VAS scores for back and leg pain had decreased to $1.2 \pm 1.3$ and $2.4 \pm 1.0$, respectively (Table 2 ). In both groups, the improvement in pain VAS on POD 1 and POD 60 indicated the efficacy of both approaches for back and leg pain. There was no conversion to surgery in any of the two groups.

The prevalence of axillary HLD (18 cases, 90\%) was higher than that of shoulder HLD (two cases, $10 \%$ ) in group B. The prevalence of central HLD was higher in group A (10 cases, $50 \%$ ) than in group B (eight cases, $40 \% ; p>0.05$ ). Two cases $(10 \%)$ of high grade migration were treated with SNRB, and one case $(5 \%)$ was treated with additional PCB $(p>0.05)$. The discs were migrated upward or downward by up to $6.4 \mathrm{~mm}$. Disc location, type, and migration were not significantly different between groups.

\section{DISCUSSION}

The psoas compartment is the space located between the psoas muscle and quadrates lumborum, and is surrounded by

Table 2. Clinical comparisons between SNRB+MBB and SNRB+ PCB groups

\begin{tabular}{llccc}
\hline & & SNRB+MBB & SNRB+PCB & $p$-value \\
\hline \multirow{2}{*}{ Pre-injection } & VAS Back & $7.7 \pm 1.0$ & $7.7 \pm 1.4$ & \\
& VAS Leg & $6.8 \pm 1.3$ & $6.5 \pm 1.5$ & $>0.05$ \\
POD 1 & VAS Back & $3.9 \pm 1.2$ & $2.9 \pm 2.3$ & $>0.05$ \\
& VAS Leg & $1.8 \pm 1.0$ & $2.3 \pm 1.3$ & \\
POD 60 & VAS Back & $2.3 \pm 1.2$ & $1.2 \pm 1.3$ & $>0.05$ \\
& VAS Leg & $1.3 \pm 1.2$ & $2.4 \pm 1.0$ & \\
\hline
\end{tabular}

SNRB: spinal nerve root block; MBB: median branch block; PCB: psoas compartment block; VAS: visual analogue scale; POD 1: post-operative day 1; POD 60: post-operative day 60 
the psoas muscle and its fascia in the front, and by the lumbar, ligaments, muscle, and quadrates lumborum in the back ${ }^{1}$. The lumbar plexus, the ventral ramus of the sacral plexus, the iliohypogastric nerve, the ilioinguinal nerve, the genitor femoral nerve, the lateral femoral cutaneous nerve, the femoral nerve, the obturator nerve, and parts of the sciatic nerve pass through this compartment ${ }^{1}$. A PCB is generally performed at the L3 or L4 disc and serves to block the lateral femoral cutaneous nerve, femoral nerve, and obturator nerve, and is therefore also known as the posterior lumbar plexus block ${ }^{8}$. PCB usually involves injection of more than $10 \mathrm{~mL}$, which can create hydrostatic pressure, resulting in adhesiolysis of pathogenic site. The iliohypogastric, ilioguinal, and genitofemoral nerve can be further blocked if the drug expands caudally within the fascia". The pathophysiology of HLD is considered to be multifactorial, and may involve myofasciopathy, which can be caused by compression of the psoas muscle. PCB may offer a conservative treatment for this condition, although the evidence therefore is still insufficient ${ }^{10)}$.

Chayen et al. first reported the use of PCB in pelvic joint and lower thigh surgery in $1976^{2}$. Dangatia and Naesh reported changes in the cross-sectional area of the psoas major muscle in unilateral sciatica caused by disc herniation ${ }^{4}$. Thus, the herniated disc not only compresses the neural component, but also affects the psoas muscle component. Treatment of the psoas muscle can thus be used to treat discogenic pain ${ }^{5)}$.

We show that both SNRBs+MBB (group A) and SNRBs+ PCB approaches (group B) were effective in HLD patients, with statistically significant effects. Both procedures showed a large reduction in the VAS scores in POD 1, but the VAS score again increased gradually. Group B showed superior effectiveness at POD 1, although the difference between the approaches was not statistically significant. Group A showed slightly higher efficacy on POD 60, but this difference was also not statistically significant.

PCB can be performed blind, or under ultrasound or fluoroscopic guidance. When ultrasound and fluoroscopy equipment are not available, the physician can perform a blind PCB, which, although it saves cost and time, poses a risk of peritoneal perforation, vascular injury, and inaccuracy. Fluoroscopic-guided PCB ensures accuracy in positioning the needle tip, but presents a radiologic risk. Ultrasound-guided PCB has many advantages, in that it improves accuracy, avoids radiological hazards, and provides continuous images; however, it involves relatively high medical costs. Despite this drawback, ultrasoundguided PCB is expected to become the mainstay of this procedure.

The amount of steroid injection is not standardized, but $40-80 \mathrm{mg}$ of triamcinolone diacetinude or $4-8 \mathrm{mg}$ of dexamethasone sodium phosphates is generally used when performing the epidural block. However, in the PCB, the muscle and fascia are located closely, and the steroid injection can result in muscle atrophy. Thus, $0.2 \%$ of lidocaine injection is added for this procedure. Several cases of complications of PCB block have been reported; these included abscesses and hematoma, and a psoas muscle abscess is considered an indication for surgical intervention. Lansdown et al. reported abscess formation in sub-optimally controlled diabetes mellitus ${ }^{6}$, because this space is highly vascularized and contains many nerves ${ }^{11)}$. If patients have accompanying diabetes mellitus, immunocompromising conditions, or are taking antiplatelet agents, the physician should consider the appropriate treatment carefully.

Although there was no statistically significant difference between the PCB and conventional approach, it may be a treatment option for uncontrollable pain, and for low back pain suspected to be of mixed muscle origin. Because PCB carries a risk of infection (e.g., abscess formation) and hematoma, which can mimic underlying malignancy, careful history taking and neurogenic examination, as well as radiological confirmation are essentia ${ }^{1)}$.

\section{CONCLUSION}

In comparison with conventional SNRB, the use of additional PCB showed a marked, although not statistically significant decrease in the VAS scores at POD 1 and POD 60. Unless an absolute indication for surgery is present, $\mathrm{PCB}$ is an option for pain management. The guidelines for PCB are not yet established; further investigation in a larger study is needed. Because some complications have already been reported, careful patient selection is essential.

\section{REFERENCES}

1. Bogduk N, Pearcy M, Hadfield G: Anatomy and biomechanics of psoas major. Clin Biomech 7:109-119, 1992

2. Chayen D, Nathan H, Chayen M: The psoas compartment block. Anesthesiology 45:95-99, 1976

3. Chelly JE, Casati A, Al-Samsam T, Coupe K, Criswell A, Tucker $\mathrm{J}$ : Continuous lumbar plexus block for acute postoperative pain management after open reduction and internal fixation of acetabular fractures. J Orthop Trauma 17:362-367, 2003

4. Dangaria TR, Naesh O: Changes in cross-sectional area of psoas major muscle in unilateral sciatica caused by disc herniation. Spine 23:928-931, 1998

5. Kim HY, Park JW, Park SY, Moon JY, Shin JH, Park SH: Psoas compartment blockade in a laterally herniated disc compressing the psoas muscle -a case report. Korean J Pain 25:116-120, 2012

6. Lansdown AJ, Downing A, Roberts AW, Martin D: Psoas abscess formation in suboptimally controlled diabetes mellitus. Case Rep Med 2011:249325, 2011

7. Lee WJ, Sung NS, Kim C: Effect of psoas compartment block in low extremity pain from stomach cancer: a case report. J Korean Pain Soc 5:113-116, 1992

8. Mannion S: Psoas compartment block. Contin Educ Anaesth Crit Care Pain 7:162-166, 2007 
9. Torres GM, Cernigliaro JG, Abbitt PL, Mergo PJ, Hellein VF, Fernandez S, et al.: Iliopsoas compartment: normal anatomy and pathologic processes. Radiographics 15:1285-1297, 1995

10. Travell JG, Simons DG: Myofascial pain and dysfunction: The trigger point manual. Baltimore: Lippincott, Williams \& Wilkins,
1983, pp 89-109.

11. Wada Y, Yanagihara C, Nishimura Y: Bilateral iliopsoas hematomas complicating anticoagulant therapy. Intern Med 44:641643,2005 